\title{
Relationship between nutritional habits adopted by ulcerative colitis relevant to cancer development patients at clinical remission stages and molecular-genetic parameters
}

\author{
Maya Rosman-Urbach ${ }^{1}$, Yaron Niv ${ }^{2}$, Yehudith Birk ${ }^{1}$, Sara Morgenstern ${ }^{2}$ and Betty Schwartz ${ }^{1^{*}}$ \\ ${ }^{1}$ Institute of Biochemistry, Food Science and Nutrition, Faculty of Agricultural, Food and Environmental Quality Sciences, \\ The Hebrew University of Jerusalem, Rehovot, Israel \\ ${ }^{2}$ Gastroenterology Department, Rabin Medical Center, Beilinson, Petah-Tikva, Israel
}

(Received 6 May 2005 - Revised 15 August 2005 - Accepted 19 September 2005)

\begin{abstract}
UC (ulcerative colitis) patients have an increased risk of developing colorectal cancer compared with the normal population. The cause underlying this higher risk is not fully defined but includes nutritional and environmental factors concomitant with genetic alterations. We aimed to evaluate genetic stability in the colonic tissue of UC patients in clinical remission compared with the healthy population, and to establish a possible correlation between nutritional habits and these molecular assessments. UC patients $(n$ 42) and healthy controls $(n$ 37) participated in the study. All participants were histopathologically and medically diagnosed. Participants completed five separate $7 \mathrm{~d}$ dietary records, food-frequency questionnaires and validated $24 \mathrm{~h}$ recalls for nutritional assessment. The extent of chromosome 17 loss and the calculated chromosome index was determined in colon tissue biopsies by fluorescence in situ hybridisation. Correlations between the molecular and nutritional assessments were performed using Pearson's correlation coefficients. Significant differences in the nutritional intake of total fat (65 (SD 15) v. 89 (SD 25) g), cholesterol (330 (SD 168) v. 464 (SD 177) mg), dietary fibre (32 (SD 4.7) v. 9 (SD 4) g), vitamin A (1009 (SD 209) v. 506 (SD 204) $\mu$ g), vitamin C (308 (SD 108) v. 72 (SD 53) mg) and folic acid (412 (SD $89 \mu \mathrm{g}) v .187$ (SD 107)) were recorded for UC patients compared with controls. Significant correlations were found for the consumption of different food groups and the chromosome index for chromosome 17. The results of our study suggest that the nutritional habits adopted by UC patients during clinical remission may affect key cellular components of the colonic tissue, inducing a high degree of aneuploidy and genetic instability, and probably affecting the development of colon cancer.
\end{abstract}

Inflammatory bowel disease: Ulcerative colitis: Nutritional habits: Fluorescence in situ hybridization

Ulcerative colitis (UC) is characterised by a constant inflammatory process of mostly unknown aetiology. Several studies indicate that environmental factors such as nutrition are associated with either the pathogenesis or, alternatively, the treatment of UC (Podolsky, 1991a,b; Fergus, 1993; Han et al. 1999; Hugot et al. 1999). Nutrient deficiencies in patients with UC occur via several mechanisms and may complicate the course of the disease (Han et al. 1999). Chronic long-standing inflammatory bowel disease (IBD) is well established as a predisposing condition for the development of colorectal adenocarcinoma (Potter et al. 1993). Whether nutrition plays a role in this predisposition remains to be investigated.

A parallel sequence of events exists between the development of sporadic colorectal carcinoma and the progression of IBD. In both, histological progression is a multistep process that includes genetic instability, clonal expansion, the development of dysplasia and finally carcinoma (Arends, 2000).

DNA aneuploidy and loss of the p53 tumour suppressor allele are found in both diseases. An abnormal aneuploid DNA pattern has been shown in sporadic colorectal cancer as well as in mucosal biopsies from premalignant conditions in the colon, such as adenoma and long-standing UC (Lofberg et al. 1989; 1992; Porschen et al. 1992; Rubin et al. 1992; Befrits et al. 1994; Lindberg et al. 1999; Pohl et al. 2000). Nonetheless, differences are also evident and indicate that loss of the p53 allele may occur earlier during neoplastic progression in UC than in sporadic colorectal cancer, in which it usually occurs late in the progression from adenoma to carcinoma (Hussain et al. 2000). In this regard, we have recently demonstrated (Rosman-Urbach et al. 2004) that there are, in UC patients, significant changes in chromosome 17 and p53 copy number, lower levels of serum p53 protein, and significant direct correlations for the duration of UC, levels of p53 in the serum and extent of aneuploidy, all measurements directly correlated with risk factors for colorectal cancer.

A plethora of experimental and epidemiological data demonstrates the potentially key role of nutritional factors in the aetiology of colorectal cancer (Lipkin et al. 1999). These studies indicate that the risk of developing colorectal neoplasia is strongly affected by the intake of fat, red meat, dietary fibre (Willett et al. 1990; Howe et al. 1992; Giovannucci et al. 1994; Lubin et al. 1997), sugars (Slatterry et al. 
1997a), folate (Brentnall et al. 1994), fruits and vegetables (Steinmetz \& Potter, 1991), as well as of other dietary factors (Potter et al. 1993). Do, then, nutritional factors affect the predisposition of UC patients to develop preneoplastic and neoplastic stages?

The present study was designed to evaluate the nutrient consumption and nutritional habits of UC patients in clinical remission and to correlate these assessments to molecular parameters that indicate genetic instability, such as the extent of cellular colonic aneuploidy and the state of chromosome 17.

\section{Methods}

Subjects

For the present study, subjects were selected from patients of the Gastroenterology Department, Rabin Medical Center, Beilinson Campus, Petah Tikva, Israel. The study was reviewed and approved by the Helsinki committee of the Rabin Medical Center, and informed written consent was obtained from each subject. Before entry into the study, all subjects were screened by medical history, physical examination and blood tests.

The study group consisted of forty-two patients (seventeen women and twenty-five men) with UC. The average age of the UC group was 55 years, with a range of 29-68 years. Their mean known disease duration was 20.6 (SD 8) years (range 11-35 years). The diagnosis of colitis was established by colonoscopy, histopathology and bowel X-ray study according to Morson's criteria (Morson, 1995). In twenty-six patients, pancolitis was diagnosed, and six had left-sided colitis. Six of the patients with UC were diagnosed with primary sclerosing cholangitis by cholangiographical and histological criteria. Four of the patients with UC exhibited dysplasiaassociated lesions and masses $(3-5 \mathrm{~cm})$ with high-grade dysplasia in the distal colon and rectum. All patients with UC had been in clinical remission from the acute phase of their disease (post-relapse stage) for less than 1 year but had suffered at least two acute attacks of UC in their medical history and were receiving preventive maintenance therapy with 5 -aminosalicylic acid. Specifically excluded were those who were thought to require intestinal surgery up to 3 months after study entry, patients with an ileostomy, colostomy or ileal pouch, patients with laboratory-confirmed intestinal infection and pregnant women.

The control group consisted of thirty-seven healthy volunteers (eighteen women and nineteen men) who had undergone colonoscopy at the gastroenterology department of the Rabin Medical Center as part of a general screening programme for colorectal cancer (CRC) and who had been diagnosed as clinically healthy. The average age of the control group was 58 years, with a range of 31-72 years. The control group was elected in order to match the age and gender distribution of the study group.

\section{Histopathological analysis of biopsy samples}

Between six and eight biopsy specimens from each UC patient and one or two biopsy specimens from each control were obtained from the colonic mucosa at colonoscopy, fixed in buffered formaldehyde and embedded in paraffin. Representative sections of each biopsy were first stained with haematoxy- lin and eosin and evaluated histopathologically. For each biopsy sample, the histopathological lesions of interest were first identified on routinely stained slides. Serial sections of lesions of interest for each biopsy were used for fluorescence in situ hybridization (FISH) analysis.

Fluorescence in situ hybridization analysis in biopsy samples

Paraffin sections from the subjects were processed for FISH analysis. We have recently described in detail methods for proteolysis, hybridisation and signal detection in FISH analyses of colonic sections (Rosman-Urbach et al. 2004).

\section{Dietary assessments}

The nutritional assessments were conducted over a period of 6 months. Three different methods of calculating daily food intake were used for each volunteer: a food-frequency questionnaire at initial screening, a $7 \mathrm{~d}$ food diary and $24 \mathrm{~h}$ recall. A trained registered dietician (M. R.-U.) performed all the dietary assessments.

The subjects attending the clinic answered the foodfrequency questionnaire and concomitantly received a packet of forms and instructions that enabled them to record a $7 \mathrm{~d}$ food diary. The packet included 'Food Diary Instructions', a complete sample day and seven blank forms. The instructions included statements on how to complete each item on the form and definitions of possible responses. The forms had a blank space for the subject's name at the top and for the date, month and year of the day being recorded. There were forty-eight lines on which to record the foods consumed each day. The first column for each line identified the meal at which the food was consumed (breakfast, lunch, dinner, snack), and the second column provided space for the specific food item. The ensuing columns provided spaces for the number of servings of each item, the place of preparation (home, work, restaurant, store) and the method of preparation (fried, sautéed, baked, broiled, microwaved, steamed, boiled, raw, uncooked, other), and to indicate whether fat was added (e.g. margarine on bread). A check box was included at the bottom of each form to indicate whether another form was needed to record the foods for that day. The subjects filled the forms on five different occasions during the 6 months of the study.

Nutritional analysis of all the data was based on food composition tables provided by the Israeli Health Administration (1996a), and specific software was developed to transform the frequencies of consumption of food items into nutrient intake data. In addition, the data were evaluated from food exchange lists for normal diets (Israeli Health Administration, $1996 \mathrm{~b}$ ). Instead of a detailed computation of the diets, these calculations evaluated consumption using an appropriate food-grouping system, which implicated grouping together foods of similar nutritive value. The use of food groups is based on the assumption that eating appropriate amounts of foods in each group will provide proper amounts of protein, minerals and vitamins. The dietary assessment was based on standard methodological formulas for pooling dietary data and analyses (Zeman \& Ney, 1988; Baeton, 1994; Friedenreich, 1994). 


\section{Anthropometric measurements}

The BMI was calculated for each subject. Total daily energy expenditure was calculated using the Harris and Benedict equations (Zeman \& Ney, 1988).

\section{Statistical analysis}

Reporting accuracy of the three intake methods was analysed by multivariate repeated-measures ANOVA. The various parameters were compared across the three subject groups by ANOVA. Associations between the extent of diploidy and other parameters were calculated and expressed by the Pearson correlation coefficient method. Statistical analyses were conducted using SAS software (version 6; SAS Institute, Cary, NC, USA).

\section{Results}

There were no statistical differences between the two groups, in terms of either age or anthropometrical parameters (Table 1).

All participants completed the data for all dietary questionnaires provided. In the present study, there was no significant difference between the three dietary assessment methodologies used (data not shown). Analyses of daily consumption of nutritional elements indicated statistically significant differences between UC and controls (Table 2). UC patients consumed significantly less dietary fibre, $\mathrm{Ca}$, vitamin A, vitamin $\mathrm{C}$ and folic acid than controls $(P=0.0001)$ but more fat $(P=0.0002)$ and cholesterol $(P=0.018)$; nonetheless, energy consumption (kcal) was similar for both groups.

To assess potential differences in the consumption of certain foods between UC patients and the healthy control group, the nutritional data were analysed using food exchange groups. Fig. 1 summarises the daily intake results calculated according to the exchange groups and shows statistically significant differences between UC and controls for vegetable protein (legumes), animal protein, vegetables, fruits, milk, fats, extras $(P=0.0001)$ and cereals $(P=0.002)$. Daily fluid intake is illustrated in Fig. 2. Whereas UC patients consumed less water and other non-caffeine-containing liquids than controls, they consumed more coffee and tea $(P=0 \cdot 0001)$.

One experienced pathologist (S. M.) diagnosed each of the sections obtained from the different biopsies. To check for interobserver reproducibility, two to four adjacent serial sections were blindly and independently evaluated by two histopathologists for different grades of dysplasia according to standard criteria for comparison (Riddell et al. 1983), with a value of 0.64 , i.e. substantial agreement (Riddell et al. 1983). Intraobserver agreement (S. M.) after 6 months was similar to the first evaluation $(0 \cdot 82)$

FISH analyses were performed as previously described (Rosman-Urbach et al. 2004). Briefly, the number of signals seen as fluorescent spots on a minimum of 100 non-overlapping interphase nuclei with intact morphology in a given area was counted using previously described criteria (Dhingra et al. 1994). A minimum of five randomly chosen areas was counted on each slide from each cell block. We calculated the polysomy index, which represented the fraction of cells showing more than two signals per cell, the monosomy index, representing the fraction of cells showing one signal per cell, and the chromosome index $(\mathrm{CI})$, the value obtained from the total number of signal spots per number of nuclei counted (including nuclei with no signal spots). Polysomy index, monosomy index and CI were evaluated by FISH analysis of the centromere of chromosome 17. The analyses did not consider cells exhibiting no fluorescence spots. Normal human lymphocytes stimulated with phytohaemagglutinin for $72 \mathrm{~h}$ were used as controls to test the probe's intrinsic hybridisation efficacy. CI has previously been demonstrated to accurately reflect the average copy number of chromosomes in intact nuclei (Dhingra et al. 1994). The CI in UC patients $(0.95 \mathrm{SD} 0.07)$ was significantly lower than in controls (1.23 SD 0.08; $P<0.01)$.

To examine whether there was a correlation between specific nutritional intake and genomic instability, a correlation analysis with the Pearson correlation coefficients for dependent variables was performed (the described variable elected being CI). Table 3 emphasises a strong negative correlation between daily dietary fat intake and CI; i.e. as fat intake increased, CI decreased. A similar negative correlation was observed between percentage energy from fat and CI. A strong positive correlation was observed for dietary fibre, $\mathrm{Ca}$, vitamin $\mathrm{A}$, vitamin $\mathrm{C}$, folic acid and legume intake when compared with CI. Table 4 shows the correlation between food exchange groups and CI. The strongest positive correlations were found for vegetable, fruit and legume (vegetable protein) consumption.

\section{Discussion}

The study described here attempts to establish a correlation between loss of DNA patterns, precarcinogenic stages and the nutritional habits adopted by UC patients at post-relapse stages. We selected UC patients who had been suffering from the disease for a long time ( $>10$ years) and who were all diagnosed as having stages of colitis with a relatively high risk of colon cancer (Rosman-Urbach et al. 2004). We chose UC patients with long-standing disease not only for

Table 1. Anthropometric characteristics of the subjects participating in the research (Results analysed by contrast $t$ test)

\begin{tabular}{|c|c|c|c|c|c|c|c|c|c|}
\hline \multirow[b]{2}{*}{ Group } & \multirow[b]{2}{*}{$n$} & \multicolumn{2}{|c|}{ Age (years) } & \multicolumn{2}{|c|}{ Weight (kg) } & \multicolumn{2}{|c|}{ Height $(\mathrm{cm})$} & \multicolumn{2}{|c|}{$\mathrm{BMI}$} \\
\hline & & Mean & SD & Mean & SD & Mean & SD & Mean & SD \\
\hline Ulcerative colitis & 42 & $55 \cdot 0$ & $13 \cdot 2$ & $70 \cdot 3$ & $16 \cdot 9$ & $167 \cdot 1$ & 8.4 & $25 \cdot 3$ & $6 \cdot 2$ \\
\hline Control (healthy volunteers) & 37 & $58 \cdot 1$ & $12 \cdot 1$ & $73 \cdot 7$ & $16 \cdot 2$ & $165 \cdot 8$ & 8.4 & $26 \cdot 7$ & $5 \cdot 1$ \\
\hline
\end{tabular}

No statistically significant differences were obtained for any parameter. 
Table 2. Mean daily energy and nutrient consumption

(Based on $24 \mathrm{~h}$ recall, food diaries and personal interviews)

\begin{tabular}{|c|c|c|c|c|}
\hline \multirow[b]{2}{*}{ Energy and nutrients } & \multicolumn{2}{|c|}{ Control } & \multicolumn{2}{|c|}{ Ulcerative colitis $†$} \\
\hline & Mean & SD & Mean & SD \\
\hline Energy (kJ) & $517 \cdot 4$ & $58 \cdot 8$ & 537 & $113 \cdot 0$ \\
\hline Protein $(\mathrm{g})$ & 90 & 13 & 81 & 21 \\
\hline Protein (\% energy) & $13 \cdot 3$ & $6 \cdot 9$ & $12 \cdot 7$ & $5 \cdot 1$ \\
\hline Fat (g) & 65 & 15 & 89 & 25 \\
\hline Fat (\% energy) & 27 & 0.1 & 35 & 0.1 \\
\hline SFA (\% energy) & $9 \cdot 9$ & 0.8 & $11 \cdot 3$ & 2.99 \\
\hline MUFA (\% energy) & $10 \cdot 5$ & 2.5 & $11 \cdot 3$ & 4.5 \\
\hline Total PUFA (\% energy) & $6 \cdot 8$ & 0.9 & $7 \cdot 1$ & $4 \cdot 2$ \\
\hline n-6 PUFA (\% energy) & 5.4 & 0.6 & $6 \cdot 6$ & 0.7 \\
\hline$n$-3 PUFA (\% energy) & 1.4 & 0.1 & 0.8 & 0.09 \\
\hline Total carbohydrates (g) & 191 & 41 & 225 & 44 \\
\hline Carbohydrates (\% energy) & $50 \cdot 6$ & $9 \cdot 1$ & $59 \cdot 8$ & $12 \cdot 9$ \\
\hline Fructose (g) & 25 & 11 & 29 & 14 \\
\hline Lactose $(\mathrm{g})$ & 12 & 8 & 14 & 9 \\
\hline Sucrose (g) & 61 & 9 & 91 & 14 \\
\hline Cholesterol (mg) & 330 & 168 & 464 & 177 \\
\hline Dietary fibre (g) & 32 & 4.7 & 9 & 4 \\
\hline $\mathrm{Ca}(\mathrm{mg})$ & 775 & 223 & 469 & 225 \\
\hline $\mathrm{Fe}(\mathrm{mg})$ & 15 & 5 & 19 & 7 \\
\hline Vitamin $\mathrm{A}(\mu \mathrm{g})$ & 1009 & 209 & 506 & 204 \\
\hline Vitamin E (mg) & 13.6 & 6 & 13.4 & 4 \\
\hline Vitamin C (mg) & 308 & 108 & 72 & 53 \\
\hline Legumes $(\mathrm{g})$ & 21.9 & 6.9 & $11 \cdot 2$ & $6 \cdot 6$ \\
\hline Folic acid $(\mu \mathrm{g})$ & 412 & 89 & 182 & 107 \\
\hline
\end{tabular}

Energy consumption was similar for both study groups. There are significant differences between the ulcerative colitis and control groups in terms of the daily consumption of nutritional elements. Patients with ulcerative colitis consumed less dietary fibre, $\mathrm{Ca}$, vitamin $\mathrm{A}$, vitamin $\mathrm{C}$, folic acid and legumes $(P=0.0001)$ but more fat $(P=0.0002)$ and cholesterol $(P=0.018)$.

the cancer risk factor, but also to deal with a population who had for a long time adopted a diet that they thought would relieve the symptoms of their disease. To obtain a reliable prospective FISH evaluation of specimens from UC patients, the tissues utilised in the present study were obtained from patients in clinical remission from the disease with no signs of active inflammation. In the present study, we elected to use as the control group an asymptomatic population who had come to the gastroenterology clinic for colorectal cancer

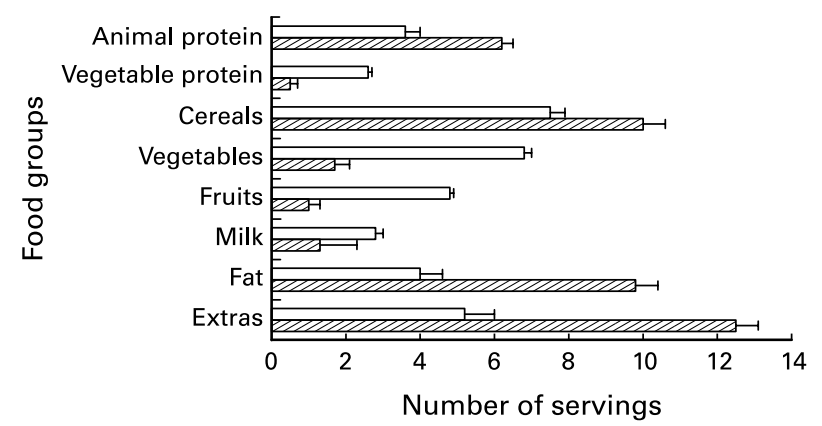

Fig. 1. Food consumption evaluated by food-exchange groups. The graph illustrates daily intake calculations (means with their standard errors) based on food-exchange lists for a normal diet. Statistically significant differences between the study groups were obtained for all food groups analysed: Animal protein (meat, poultry, fish, eggs), Vegetable protein (legumes, soya products), Vegetables, Fruits, Milk, Fats and Extras (sugars and simple carbohydrates found in jams, biscuits, cakes, chocolate, etc.) $(P=0.0001)$, and Cereals $(P=0.002)$. $\square$, Control; 证, ulcerative colitis.

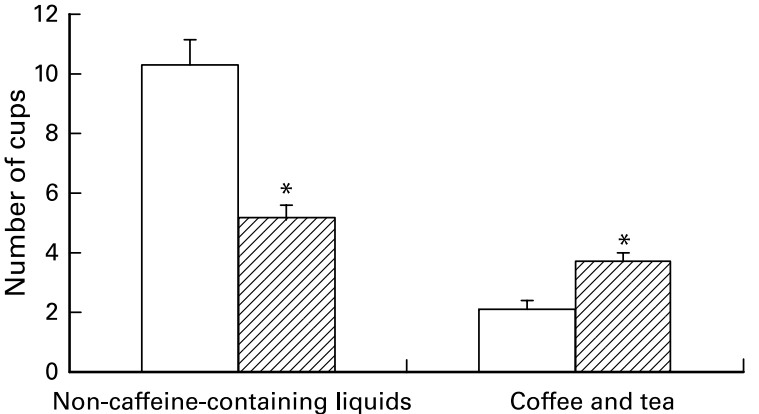

Fig. 2. Mean daily fluid and coffee consumption. Summary of daily fluid intake (means and their standard errors). Non-caffeine-containing liquids include water, juices, herb tea and some soft drinks. A statistically significant difference $(P=0.0001)$ was found for controls compared with patients with ulcerative colitis. $\square$, Control; $\mathbb{Z}$, ulcerative colitis.

screening and had had a normal colonoscopy. The medical and histopathological examinations confirmed that the control group did not suffer from bowel-related disorders.

Our first aim was to perform extensive food- and nutrientconsumption assessments in the UC patient group and to compare these with those of a healthy control group. To this end, nutrient-consumption and food-intake habits were obtained and calculated from records and interviews according to well-established methods.

Nutrient-consumption assessments revealed that UC patients at post-relapse stages consume less dietary fibre, $\mathrm{Ca}$, vitamin $\mathrm{A}$, vitamin $\mathrm{C}$ and folic acid than their control counterparts. These findings are at odds with previous studies reporting nutritional deficiencies in UC patients, mainly owing to decreased consumption (Han et al. 1999). These parameters were further evaluated according to the exchange-list methodology in relation to a normal diet. These analyses clearly demonstrated significant differences between UC patients and controls in the intake of all food groups. UC patients consumed fewer legumes, vegetables, fruit and milk products, but more meat, cereals, fat and simple carbohydrates ('Extras'). Our findings agree with previous reports that carbohydrate consumption is significantly higher in UC patients than in healthy controls, and that UC patients also consume more total protein and less fruit than controls (Tragnone et al. 1995).

Based on the food-intake analyses, nutrient consumption by the UC group exemplifies a characteristic high-risk diet for the development of colon cancer. For example, the increased consumption of meat in the UC group is believed to be one of the risk factors for the development of colon cancer (Geraldine et al. 1988; Lipkin et al. 1999). In addition, UC patients consume small amounts of foods from the vegetable protein group, and legume consumption, especially of soya and its derivatives, has been found to be cancer-preventive (Ritenbaugh, 2000).

Ryder et al. (1998) have proposed that the common expression of galactose $\beta$ 1,3-N-acetyl galactosamine $\alpha$ (Thomsen-Friedenreich antigen) by hyperplastic and neoplastic epithelia may be functionally important because it allows an interaction with mitogenic dietary lectins. Legumes contain high concentration of lectins, and the question arises of whether the lower legume consumption measured in UC 
Table 3. Correlation between nutritional elements and calculated chromosome index in the group with ulcerative colitis

\begin{tabular}{lcc}
\hline Nutritional element & Pearson correlation coefficients $(r)$ & Significance from zero $(P)$ \\
\hline Dietary fibre $(\mathrm{g})$ & 0.79 & 0.0001 \\
Vitamin C (mg) & 0.62 & 0.0001 \\
Folic acid $(\mathrm{mg})$ & 0.72 & 0.0001 \\
Legumes $(\mathrm{g})$ & 0.60 & 0.0001 \\
Vitamin A (mg) & 0.57 & 0.0001 \\
\% Fat of calories & -0.54 & 0.0001 \\
Ca $(\mathrm{mg})$ & 0.42 & 0.0032 \\
Fat $(\mathrm{g})$ & -0.51 & 0.0039 \\
\hline
\end{tabular}

A negative correlation was found for percentage fat of calories $(r=-0.54, P=0.0001)$ and fat $(r=-0.51$, $P=0.0039)$. All other elements show strong positive correlations with highly statistical significance $(P=0.0001)$

Table 4. Correlation between food groups (calculated by food exchanges) and chromosome index

\begin{tabular}{lcc}
\hline Food groups & $\begin{array}{c}\text { Pearson correlation } \\
\text { coefficients }(r)\end{array}$ & $\begin{array}{c}\text { Significance } \\
\text { from zero }(P)\end{array}$ \\
\hline Fruits & 0.77 & 0.0001 \\
Vegetables & 0.76 & 0.0001 \\
Vegetable protein & 0.66 & 0.0001 \\
Extras & -0.57 & 0.0001 \\
Fat & -0.56 & 0.0001 \\
Milk & 0.49 & 0.0005 \\
Animal protein & -0.45 & 0.0014 \\
Cereals & -0.39 & 0.007 \\
\hline
\end{tabular}

Half of the food groups' analyses (Fruits, Vegetables, Vegetable protein, Milk) show statistically significant positive correlations and half statistically significant negative correlations.

patients, which is translated into lower lectin levels, might protect these patients from CRC. The answer is not straightforward as previous studies by $\mathrm{Yu}$ et al. (2001) indicate that two dietary Thomsen-Friedenreich antigen-binding lectins induce opposite effects on the proliferation of human colon cancer cells. Whereas peanut (Arachis hypogea) and edible mushroom (Agaricus bisporus) produced marked stimulatory effects on human intestinal epithelial cell proliferation, two other known dietary Thomsen-Friedenreich antigen-binding lectins - jacalin (Artocarpus integrifolia) and amaranth lectin (Amaranthus caudatus) - produced a dose-dependent inhibition of proliferation in HT29 human colon cancer cells. Therefore, the types of lectin consumed may determine the proliferation of colonic cells.

Our dietary assessments showed that UC patients consumed low amounts of fibre, vegetables and fruits, behaviour that is directly related to an increased risk of colorectal cancer in the general population (Howe et al. 1992; Adlercreutz et al. 1995; Morson, 1995; Giovannucci, 1999; Lubin et al. 1997). The control group ingested 32 (SD 4.7) g fibre daily, whereas the UC group consumed significantly less fibre (9 (SD 4) $\mathrm{g}$ daily). The intake of dietary fibre in the normal Israeli population is relatively high and ranges from 27.4 (SD 11.9) $\mathrm{g}$ to 34.4 (SD 14.8) g daily depending on the ethnic group of country of origin (Lubin et al. 2003). The control group tended to consume fibre within the higher range since they had a family history of colon cancer and might be consuming a relatively healthy diet. In contrast, UC patients tended to avoid fibre in order to reduce their symptoms, and this translated into the ingestion of significantly lower quantities of fibre daily.

Various studies suggest that a high consumption of fat and meat is an important dietary risk factor for the development of colorectal cancer (Slattery et al. 1997b; Singh \& Fraser, 1998), even in low-risk populations (Slattery et al. 1997b). These foods are thought to be 'aggressive' factors, which may trigger the pathogenesis of UC itself and concomitantly increase the risk of developing or aggravating UC (Reif et al. 1997). We showed that UC patients consumed high amounts of sugars and simple carbohydrates, which are also associated with an increased risk of colorectal cancer among younger men (Giovanna et al. 1998). The most significant carbohydrate consumed was sucrose, supporting previous findings for Israeli IBD patients (Reif et al. 1997).

The low intake of $\mathrm{Ca}$ in the UC group is probably associated with their low consumption of dairy products during all phases of the disease. This nutritional deficiency is one of the risk factors in the development of osteoporosis, and the prevalence of low bone mass in patients with UC has been reported to be more than $50 \%$ (Andreassen et al. 1997). UC patients suffer from an increased degradation of type I collagen (Silvennoinen et al. 1996) and altered bone metabolism (Bischoff et al. 1997). Other risk factors for the development of low bone mass in patients with UC include the general risk factors for osteoporosis, the presence of inflammation and the use of corticosteroids and other pharmaceuticals (Cowan et al. 1997; Valentine \& Sninsky, 1999). Furthermore, the low consumption of $\mathrm{Ca}$ has been found to be associated with an increased risk of developing colon tumours (Singh \& Fraser, 1998).

A low consumption of vitamin $\mathrm{A}$, as found in the present study (Table 2), is typical of UC patients (Bousvaros et al. 1998) and is also considered to be a high-risk factor for developing colorectal cancer, owing to the antioxidant role played by this vitamin (Niles, 2000). We found the folic acid intake of UC patients to be lower than the RDA and lower than that of controls. There is growing evidence that folic acid deficiency is associated with a variety of human malignancies, including colorectal cancer. Populations consuming a diet low in folic acid have a high incidence of adenomatous polyps in the colon (Giovannuci et al. 1993), and daily supplementation with folic acid may protect against the development of neoplasia in UC. 
The intake of total fat was higher in the UC population than the control population, supporting previous findings in Israeli IBD patients (Reif et al. 1997). Notably, the consumption of $n$-6 PUFA was higher, but the consumption of $n-3$ PUFA was lower, in IBD. Diets with a specific fat composition may influence inflammatory and immunological events (Gassull et al. 2005). The consumption of $n-6$ PUFA greatly exceeds that of $n-3$ PUFA. The $n-6$ PUFA arachidonic acid gives rise to the eicosanoid family of inflammatory mediators (prostaglandins, leukotrienes and related metabolites) and through these regulates the activities of inflammatory cells, the production of cytokines and the various balances within the immune system. Vegetable oils such as safflower, soya, corn and sunflower oil contain high levels of the $n$-6 PUFA linoleic acid. The consumption of long-chain n-3 PUFA decreases the amount of arachidonic acid in cell membranes and therefore available for eicosanoid production. Thus, $n-3$ PUFA act as arachidonic acid antagonists.

Calder \& Grimble (2002) have proposed the likely mechanisms by which inflammatory responses can be modulated through differing ratios of $n-3$ and $n-6$ PUFA. Human inflammatory cells typically contain high proportions of arachidonic acid and low proportions of $n$-3 PUFA. The primary effect of $n$-3 PUFA is likely to be through a modulation of the amounts and types of eicosanoid made. Feeding sources of $n-3$ fatty acids results in a partial replacement of arachidonic acid in inflammatory cell membranes by EPA. This change leads to a decreased production of arachidonic acid-derived mediators that regulate the activities of inflammatory cells and the production of cytokines. $n-3$ fatty acids thereby act as arachidonic acid antagonists, decreasing the amount of arachidonic acid in cell membranes that is available for eicosanoid production.

As well as this direct mechanism for anti-inflammatory effects, there is increasing evidence that $n-3$ PUFA may have an indirect action mediated through TNF- $\alpha$ and NF- $\kappa \mathrm{B}$ production (Babcock et al. 2002). A direct link between NF$\kappa \mathrm{B}$ production, inflammation and cancer has been demonstrated by Greten et al. (2004). Proinflammatory cytokines trigger NF- $\kappa \mathrm{B}$ activation via the IкB kinase (IKK) complex. In an elegant work, Greten et al. (2004), using a colitis-associated cancer model, demonstrated that the deletion of IKK $\beta$ in intestinal epithelial cells led to a dramatic decrease in tumour incidence. Deleting IKK $\beta$ in myeloid cells, however, also resulted in a significant decrease in tumour size. They therefore concluded that specific inactivation of the IKK/NF-кB pathway in two different cell types attenuated the formation of inflammation-associated tumours and directly linked inflammation to cancer. We can thus conclude that a nutritional control of inflammation status may directly affect colitis-associated cancer.

In the present study, UC patients consumed less water than healthy controls but more coffee, tea and other caffeine-containing liquids. In this regard, Lubin et al. (1997) have reported that a high water intake is significantly and negatively associated with the development of colorectal adenoma. Furthermore, high coffee consumption has been correlated with an increased risk of colorectal cancer in some studies (Potter et al. 1993, Terryl et al. 1998).

Cumulatively, the nutritional evidence provided in this study confirms previous findings in that the dietary habits adopted by UC patients concur with those of populations who are at high risk of developing colorectal cancer.

We hypothesised that if the nutritional intake of UC patients correlated to a higher risk of colorectal tumorigenesis, the molecular features typical of pre-cancer or proper neoplastic stages might be evident. We therefore performed molecular analyses in these populations and correlated them to the nutritional assessments of their food intake.

The calculated CI of chromosome 17 was significantly lower in the colonic tissues of UC patients than in healthy controls, confirming a greater degree of aneuploidy in tissues from patients with UC. Aneuploidy has been described in mucosal biopsies from premalignant conditions in the colon, such as UC dysplasia (Ahnen, 1992; Markowitz et al. 1997), and long-standing UC (Levins et al. 1991). A 10-year follow-up study (Befrits et al. 1994) revealed that DNA aneuploidy in chronic UC appeared to be stable and might precede histological dysplasia by many years. It also appeared to be an additional marker for detecting neoplastic transformation in UC patients (Levins et al. 1991; Lofberg et al. 1992; Porshen et al. 1992; Rubin et al. 1992; Lindberg et al. 1999) that correlates with histological studies.

To our knowledge, this is the first time that nutritional and molecular assessments have been correlated. Our results show a strong correlation between daily nutrient intake and CI level. We found a strong negative correlation between fat intake and $\mathrm{CI}$, whereas a strong positive correlation was observed with the intake of dietary fibre, $\mathrm{Ca}$, vitamin $\mathrm{A}$, vitamin $\mathrm{C}$ and folic acid. Food consumption evaluated by food-exchange lists also showed a statistically significant correlation with respect to the intake of all food groups. A positive correlation was found between $\mathrm{CI}$ and the consumption of fruits, vegetables, legumes, vegetable protein and milk. Negative correlations between diploidy and food groups included the consumption of sugars and simple carbohydrates ('Extras'), meat and cereals.

In conclusion, the results of our study suggest that the food intake of UC patients at all disease stages may affect key cellular components in the colonic tissue, cause genomic instability and aneuploidy, and enhance the predisposition of these patients to colon cancer. The phenomenon described of a closed cycle in which the disease dictates a diet that aggravates the disease should be addressed in future studies.

\section{References}

Adlercreutz CH, Goldin BR, Gorbach SL, et al. (1995) Soybean phytoestrogen intake and cancer risk. J Nutr 34, 757s-767s.

Ahnen DJ (1992) Abnormal DNA content as a biomarker of large bowel cancer risk and prognosis. J Cell Biochem Suppl 166, $143-150$.

Andreassen H, Rungby J, Dahlerup JF \& Mosekilde L (1997) Inflammatory bowel disease osteoporosis. Scand J Gastroenterol 32, $1247-1255$.

Arends JW (2000) Molecular interactions in the Vogelstein model of colorectal carcinoma. J Pathol 190, 412-416.

Babcock TA, Helton WS, Hong D \& Espat NJ (2002) Omega-3 fatty acid lipid emulsion reduces LPS-stimulated macrophage TNFalpha production. Surg Infect 3, 145-149.

Baeton GH (1994) Approaches to analyses of dietary data: relationship between planned analyses and choice of methodology. Am J Clin Nutr 59, 253s-261s. 
Befrits R, Hammarberg C, Rubio C, Jaramillo E \& Tribukait B (1994) DNA aneuploidy and histologic dysplasia in long-standing ulcerative colitis. A 10-year follow-up study. Dis Colon Rectum 37, 313-319.

Bischoff SC, Hermann A, Goke M, Manns MP, von zur Muhlen A \& Brabant G (1997) Altered bone metabolism in inflammatory bowel disease. Am J Gastroenterol 92, 1157-1163.

Bousvaros A, Zurakowski D, Duggan C, Law R, Rifai N, Golderberg NE \& Leichtner AM (1998) Vitamins A and E serum levels in children and young adults with inflammatory bowel disease: effect of disease activity. J Pediatr Gastroenterol Nutr 26, 129-135.

Calder PC \& Grimble RF (2002) Polyunsaturated fatty acids, inflammation and immunity. Eur J Clin Nutr 56, Suppl. 3, S14-S19.

Cowan FJ, Warner JT, Dunstan FD, Evans WD, Gregory JW \& Jenkins HR (1997) Inflammatory bowel disease and predisposition to osteopenia. Arch Dis Child 76, 325-329.

Dhingra K, Sneige N, Pandita TK, Johnston DA, Lee JS, Emami K, Hortobagyi GN \& Hittelman WN (1994) Quantitative analysis of chromosome in situ hybridization signal in paraffin-embedded tissue sections. Cytometry 16, 100-112.

Fergus S (1993) Pathogenesis of ulcerative colitis. Lancet 342, 407-412.

Friedenreich AM (1994) Methodologic issues for pooling dietary data. Am J Clin Nutr 59, 251s-252s.

Gassull MA, Mane J, Pedrosa E \& Cabre E (2005) Macronutrients and bioactive molecules: is there a specific role in the management of inflammatory bowel disease? J Parenter Enteral Nutr 29, Suppl. 4, S179-S183.

Geraldine P, Ginette P, Sylviane T \& Denis E (1988) Effects of meat (beef, chicken and bacon) on rat colon carcinogenesis. Nutr Cancer 32, 165-173.

Giovanna C, Lorella L, Domenico P, et al. (1998) A dietary trial with a short-term low-sucrose diet in an Italian population: effects on colorectal mucosal proliferation. Nutr Cancer 32, 159-164.

Giovannucci E (1999) Nutritional factors in human cancers. Adv Exp Med Biol 472, 29-42.

Giovannucci E, Rimm EB, Stampfer MJ, Colditz GA, Ascherio A \& Willett WC (1994) Intake of fat, meat and fiber in relation to risk of colon cancer in men. Cancer Res 54, 2390-2397.

Giovannucci E, Stampfer MJ, Colditz GA, Rimm EB, Trichopolous D, Rosner BA, Speizer FE \& Willet WC (1993) Folate, methionine, and alcohol intake and the risk of colorectal adenoma. $J$ Natl Cancer Inst 85, 875-884.

Greten FR, Eckmann L, Greten TF, Park JM, Li ZW, Egan LJ, Kagnoff MF \& Karin M (2004) IKKbeta links inflammation and tumorigenesis in a mouse model of colitis-associated cancer. Cell 118, 285-296.

Han PD, Burke A, Baldassano RN, Rombeau JL \& Lichtenstein GR (1999) Nutrition and inflammatory bowel disease. Gastroenterol Clin North Am 28, 423-443.

Howe GR, Benito E, Castelleto R, et al. (1992) Dietary intake of fiber and decreased risk of cancers of the colon and rectum: evidence from the combined analysis of 13 case-control studies. $J$ Natl Cancer Inst 84, 1887-1896.

Hugot JP, Zouali H, Lesage S \& Thomas G (1999) Etiology of the inflammatory bowel diseases. Int $J$ Colorectal Dis 14, 2-9.

Hussain SP, Amstad P, Raja K, et al. (2000) Increased p53 mutation load in noncancerous colon tissue from ulcerative colitis: a cancer prone chronic inflammatory disease. Cancer Res 60, 3333-3342.

Israeli Health Administration (1996a) Food Exchange Lists for Normal Diet. Jerusalem: Israeli Government Printing Office.

Israeli Health Administration (1996b) Food Tables. Jerusalem: Israeli Government Printing Office.

Levins DS, Rabinovitch PS, Haggitt RC, Blount PL, Dean PJ, Rubin CE \& Reld BJ (1991) Distribution of aneuploid cell populations in ulcerative colitis with dysplasia cancer. Gastroenterology 101, $1198-1210$.
Lindberg JO, Stenlling RB \& Rutegard JN (1999) DNA aneuploidy as a marker of premalignancy in surveillance of patients with ulcerative colitis. Br J Surg 86, 947-950.

Lipkin M, Reddy B, Newmark H \& Lamprecht SA (1999) Dietary factors in human colorectal cancer. Аппи Rev Nutr 19, 545-586.

Lofberg R, Brostrom O, Karlen P, Ost A \& Tribukait B (1992) DNA aneuploidy in ulcerative colitis: reproducibility, topographic distribution, and relation to dysplasia. Gastroenterology 102, $1149-1154$.

Lofberg R, Caspersson T, Tribukait B \& Ost A (1989) Comparative DNA analyses in longstanding colitis with aneuploidy. Gut $\mathbf{3 0}$, $1731-1736$.

Lubin F, Lusky A, Chetrit A \& Dankner R (2003) Lifestyle and ethnicity play a role in all-cause mortality. $J$ Nutr 133, 1180-1185.

Lubin F, Rozen P, Arieli B, Farbstein M, Knaani Y, Bat L \& Farbstein H (1997) Nutritional and lifestyle habits and waterfibre interaction in colorectal adenoma aetiology. Cancer Epidemiol Biomarkers Prev 6, 79-85.

Markowitz J, McKinley M, Kahn E, Stiel L, Rosa J, Grancher K \& Daum F (1997) Endoscopic screening for dysplasia and muscosal aneuploidy in adolescents and young adults with childhood onset colitis. Am J Gastroenterol 92, 2001-2006.

Morson BC (1995) Precancer and cancer in inflammatory bowel disease. Pathology 17, 173-180.

Niles RM (2000) Vitamin A and cancer. Nutrition 16, 573-576.

Podolsky DK (1991a) Inflammatory bowel disease (1). N Engl J Med 325, 928-937.

Podolsky DK (1991b) Inflammatory bowel disease (2). N Engl J Med 325, $1008-1016$.

Pohl C, Hombach A \& Kruis W (2000) Chronic inflammatory bowel disease and cancer. Hepatogastroenterology 7, 57-70.

Porschen R, Robin U, Schumacher A, Schauseil S, Borchard F, Hengels KJ \& Strohmeyer G (1992) DNA aneuploidy in Crohn's disease and ulcerative colitis: results of a comparative flow cytometric study. Gut 33, 663-667.

Potter JD, Slattery ML, Bostick RM \& Gapstur SM (1993) Colon cancer: a review of the epidemiology. Epidemiol Rev 15, 499-545.

Reif S, Klein I, Lubin F, Farbsteien A \& Gilat T (1997) Pre-illness dietary factors in inflammatory bowel disease. Gut 40, 754-760.

Riddell RH, Goldman H, Ransohoff DF, et al. (1983) Dysplasia in inflammatory bowel disease: standardized classification with provisional clinical applications. Hum Pathol 14, 931-968.

Ritenbaugh C (2000) Diet and prevention of colorectal cancer. Curr Oncol Rep 2, 225-233.

Rosman-Urbach M, Niv Y, Birk YC, Zussman Y, Sandler B, Morgenstein S \& Schwartz B (2004) A high degree of aneuploidy, loss of p53 gene and low p53-protein serum levels are detected in IBD patients. Dis Colon Rectum 47, 304-313.

Rubin CE, Haggitt RC, Burmer GC, et al. (1992) DNA aneuploidy in colonic biopsies predicts future development of dysplasma in ulcerative colitis. Gastroenterology 103, 1611-1620.

Ryder SD, Jacyna MR, Levi AJ, Rizzi PM \& Rhodes JM (1998) Peanut ingestion increases rectal proliferation in individuals with mucosal expression of peanut lectin receptor. Gastroenterology $114,44-49$.

Silvennoinen J, Risteli L, Karttunen T \& Risteli J (1996) Increased degradation of type I collagen in patients with inflammatory bowel disease. Gut 38, 223-228.

Singh PN \& Fraser GE (1998) Dietary risk factors for colon cancer in low-risk population. Am J Epidemiol 148, 761-774.

Slattery ML, Benson J \& Berry TD (1997a) Dietary sugar and colon cancer. Cancer Epidemiol Biomarkers Prev 6, 677-685.

Slattery ML, Berry TD, Potter J \& Caan B (1997b) Diet diversity, diet composition, and risk of colon cancer (United States). Cancer Causes Control 8, 872-882.

Steinmetz KA \& Potter JD (1991) Vegetables, fruit, and cancer. Epidemiol Cancer Causes Control 2, 325-357. 
Terryl JH, Joseph A, Tangera P, Pirjo P, Nea M, Mikko V, Philip RT \& Demetrius A (1998) Tea and coffee consumption and risk of colon and rectal cancer in middle aged Finnish men. Nutr Cancer 31, 41-48.

Tragnone A, Valpiani D, Miglio F, Elmi G, Bazzocchi G, Pipitone E \& Lanfranchi GA (1995) Dietary habits as risk factors for inflammatory bowel disease. Eur J Gastroenterol Hepatol 7, 47-51.

Valentine JF \& Sninsky CA (1999) Prevention and treatment of osteoporosis in patients with inflammatory bowel disease. Am J Gastroenterol 94, 878-883.
Willett WC, Stampfer MJ, Colditz GA, Rosner BA \& Speizer FE (1990) Relation of meat, fat, and fiber intake to the risk of colon cancer in a prospective study among women. $N$ Engl J Med 323, 1664-1672.

Yu LG, Milton JD, Fernig DG \& Rhodes JM (2001) Opposite effects on human colon cancer cell proliferation of two dietary ThomsenFriedenreich antigen-binding lectins. J Cell Physiol 186, 282-287.

Zeman FJ \& Ney DM (1988) Evaluating nutritional status. In Applications of Clinical Nutrition, In: pp. 18-31 [FJ Zeman and DM Ney, editors]. Englewood Cliffs, NJ: Prentice Hall. 